\title{
Analysis tandem repeats and retrotransposons of Shepherdia argentea (Pursh) Nutt.
}

\author{
Karina Bone \\ Laboratory of plant genetic engineering \\ All-Russia Research Institute of \\ Agricultural Biotechnology \\ Russian State Agrarian University - \\ Moscow Timiryazev Agricultural \\ Academy \\ Moscow, Russia \\ karinabone@mail.ru
}

\author{
Olga Razumova \\ Laboratory of applied genomics and \\ crop breeding \\ Kurchatov Genomic Center \\ All-Russia Research Institute of \\ Agricultural Biotechnology \\ razumovao@gmail.com \\ Gennady Karlov \\ All-Russia Research Institute of \\ Agricultural Biotechnology \\ Moscow, Russia \\ karlovg@gmail.com
}

\author{
Ilya Kirov \\ Laboratory of marker-assisted and \\ genomic selection of plants \\ All-Russia Research Institute of \\ Agricultural Biotechnology \\ Moscow, Russia \\ kirovez@gmail.com
}

\begin{abstract}
Buffalo berry (Shepherdia arg.) is a dioecious plant of the Elaeagnaceae family, being widely used in tradicional medicine. In our work, we carried out sequencing of two Shepherdia plants followed by repeatome annotation. Tandem repeats and mobile elements including both DNA transposons and retrotransposons have been identified, classified and their genome abundancy were predicted. Comparative analysis of the repeatomes of male and additional Shepherdia plant having unknown sex revealed differences in several repeat families.
\end{abstract}

Keywords - shepherdia arentea, satellite DNA, mobile elements,

\section{Introduction}

Buffalo berry is a dioecious plant of the Elaeagnaceae family, it is a close relative of sea buckthorn, whose homeland is North America. As well as sea buckthorn, buffalo berry is widely used in tradicional medicine, as it is a source of useful elements - in the berries of buffalo berry there is a high content of vitamin $\mathrm{C}$, carotene, catechins. Among the economically significant signs of buffalo berry, one can note such signs as the ability to enrich the soil with nitrogen due to the presence of nitrogen-fixing bacteria on the roots and resistance to drought. As well as her close relative, sea buckthorn, buffalo berry begins to bear fruit only by the age of 5 , which, together with dioeciousness, complicates its selection. In this regard, it seems relevant to study the genetics of this species, to create the basis for marker selection.

\section{Methods}

In our work, we performed Illumina sequencing of two shepherdia plants. At the first stage, we checked the quality of the obtained sequences using the Fastqc program [1].Adapters were trimmed using the Trimmomatic program [2], and then the trimmed sequences were checked again in Fastqc. After that, using the RepeatExplorer program [3], we performed a graph-based clustering and tandem repeat analysis of the sequences of two plants of Shepherdia arg. - male and unknown sex. The total number of input read pairs was: $11,134,794$ for male plant and 6,407,362 for the plant with unknown sex. In both plants, we analyzed 3 million randomly selected reads. The resulting consensus sequences of satellite repeats were compared between two plants using BLASTN program [4]. Primers were designed to detect for chromosome mapping using fluorescence in situ hybridization (FISH).

\section{Phylogenetic tree}

The reverse transcriptase domain of retrotransposon elements were extracted from the contigs assembled by RepeatExplorer using DANTE (Domain based Annotation of Transposable Elements, Neumann et al. (in press)). Sequences of retrotransposons Ty3 / Gypsy and Ty1 / Copia based on reverse transcriptase sequences were aligned by MUSCLE [5] and Neighbor-Joining tree was constructed for Ty3/Gypsy and Ty1/Copia.

\section{Results}

Using RepeatExplorer clustering of Illumina genomic reads for two Shepherdia plants tandem repeats and retrotransposons were found. A deeper bioinformatics analysis and annotation of DNA repeats of male and unknown sex shepherdia plants revealed 8 satellite repeats and 7 satellite repeats, respectively.. Among Ty1/Copia retrotransposons we found fifteen clusters corresponding to Ale subfamilies, twelve clusters corresponding to Angela/ Tork subfamilies, three clusters corresponding TAR subfamilies, seven clusters corresponding Ivana subfamilies and two clusters corresponding SIRE subfamilies. Among Ty3/Gypsy retronsposons we found eleven Athila subfamilies, three Tekay subfamilies, one Galadriel subfamily, four CRM subfamilies and sixty Reina subfamilies.

\section{ACKNOWLEDGMENT}

This work was supported by a grant from the Russian Foundation for Basic Research, agreement No. 20-016-00145 A.

\section{REFERENCES}

[1] Fastqc Version 0.11.8: https://www.bioinformatics.babraham.ac.uk/ projects/fastqc/

[2] Bolger, A. M., Lohse, M., \& Usadel, B. (2014). Trimmomatic: A flexible trimmer for Illumina Sequence Data. Bioinformatics, btu170.

[3] Novak, P., Neumann, P., Pech, J., Steinhaisl, J., Macas, J. (2013) RepeatExplorer: a Galaxy-based web server for genome-wide characterization of eukaryotic repetitive elements from next generation sequence reads. Bioinformatics 29:792-793.

[4] Zhang, J. \& Madden, T.L. (1997) "PowerBLAST: A new network BLAST application for interactive or automated sequence analysis and annotation." Genome Res. 7:649-656. PubMed

[5] Edgar, R.C. (2004) MUSCLE: multiple sequence alignment with high accuracy and high throughput.Nucleic Acids Res. 32(5):1792-1797. 